\title{
Review
}

Yuan Liu*

\section{Immunomodulatory effects of}

\section{Tim-3 and PD-1 on chronic hepatitis B virus infection}

DOI: $10.2478 / \mathrm{ii}-2018-0012$

Received February 21, 2018; accepted February 21, 2018; published online May 15, 2018

Abstract: In patients with chronic hepatitis B virus (HBV) infection, the immune cells are dysfunctional, and the immune function cannot work normally. T-cell immunoglobulin mucin-3 (Tim-3) and programmed death receptor-1 (PD-1) are overexpressed on the surface of immune cells, such as cluster of differentiation (CD) $4^{+}$, $\mathrm{CD}^{+}$T-lymphocytes, and natural killer (NK) cells. Many studies indicate that this phenomenon is closely related to the persistence, occurrence, development, and prognosis of HBV. Tim-3 and PD-1 may be used as new immune targets for the treatment of chronic hepatitis B.

Keywords: Hepatitis B virus, immunoregulation, Tim-3, PD-1

\section{Introduction}

Chronic infection with the hepatitis B virus (HBV) is prevalent worldwide. According to the World Health Organization (WHO), approximately 2 billion people in the world have been infected with $\mathrm{HBV}$, and 240 million of these patients have chronic HBV infection [1]. A total of 650,000 deaths occur annually from the complications caused by chronic HBV infection; these complications include liver failure, cirrhosis, and hepatocellular carcinoma [2]. HBV is the pathogen of chronic hepatitis B (CHB) and affects the development of the disease by inducing a series of immune responses within the infected organism. These immune responses are mediated by dendritic cells, natural killer (NK) cells, monocytes/macrophages, cluster of differentiation (CD) $8^{+}$T-lymphocytes, CD4 $4^{+}$T-lymphocytes, and other immune cells that play an important role in chronic HBV infection. Some studies have shown that in CHB patients, these immune cells have different degrees of dysfunction, but the mechanism underlying this phenomenon is poorly understood [3-6]. The expression of some immune molecules in these immune cells has recently become a research hot spot. The overexpression of T-cell immunoglobulin mucin-3 (Tim-3) and programmed death receptor-1 (PD-1) in immune cells, such as $\mathrm{CD}^{+} \mathrm{T}$-lymphocytes, $\mathrm{CD}^{+} \mathrm{T}$-lymphocytes, and NK cells, is closely related to chronic HBV infection. In this paper, the immunomodulatory effects of these immune molecules on chronic HBV infection are reviewed.

\section{Role of Tim-3}

\subsection{Overview}

Tim-3 has recently become a research hot spot in the field of chronic viral infection and tumor immunity. Tim-3 belongs to the Tim family and was first discovered by McIntire et al. [7] when studying asthma-related genes. Tim-3 is a T-cell membrane protein gene that encodes the immunoglobulin variable domain and the mucin

Department of Dermatovenereology, Tianjin Medical University General Hospital, Tianjin, China

*Correspondence: Yuan Liu, E-mail: yliutjmu@126.com 
domain. Eight Tim genes encode four proteins, namely, Tim-1, Tim-2, Tim-3, and Tim-4 in mice, whereas only Tim-1, Tim-3, and Tim-4 [7] are encoded in humans. All Tim family members are type I transmembrane glycoproteins that are found on the cell surface and include a signal sequence, the N-terminal immunoglobulin domain, the mucin domain, a transmembrane region, and an intracellular region containing tyrosine phosphorylation motif [8]. The signaling pathway composed of Tim-3 and its ligand galactoagglutinin-9 (Gal-9) plays an important role in tumorigenesis and development of chronic viral infection. Preliminary studies on the immunosuppressive effect of Tim-3 in chronic viral infections show that when the expression of Tim-3 in the peripheral blood T-lymphocytes of hepatitis $\mathrm{C}$ virus (HCV)-infected people is higher than that of healthy people, the proliferation and secretion functions of $\mathrm{CD} 4^{+}$and $\mathrm{CD} 8^{+} \mathrm{T}$-lymphocytes are partly restored when the Tim-3 signaling pathway is blocked [9]. In addition, the expression of Tim-3 on NK cells and T-lymphocytes in the peripheral blood increases when the person is infected with human immunodeficiency virus (HIV). After high-efficiency antiretroviral therapy, the expression levels of Tim-3 decrease in some immuneresponsive patients [10]. However, in tumor immunity, the expression of Tim-3 is significantly enhanced and even associated with the prognosis of the disease [11-13]. These results suggest that the expression of Tim- 3 is related to tumorigenesis and development of chronic viral infection.

\subsection{Tim-3 and chronic HBV infection}

Tim-3 plays a negative immunomodulatory role in chronic HBV infection. Rong et al. [14] found that the expression of Tim-3 on the surface of peripheral blood mononuclear cells and NK T-cells (NKT cells) in CHB patients is significantly higher than that in healthy persons; this phenomenon is evident in patients with CHB and acute-on-chronic liver failure. The findings of Ju et al. [15] are similar to those of Yong et al. In addition, the expression levels of Tim-3 on NK cells and $\mathrm{CD}^{+}$T-lymphocytes in the liver are also significantly upregulated, as confirmed by the study group using HBV-transfected NK92 cell lines and HBV transgenic mouse models. On this basis, Tim-3 antibody is used to block the Tim-3/Gal-9 signaling pathway. The cytotoxicity of NK cells is enhanced, and their secretion of interferon (IFN)-y is also increased. Wu et al. [16] showed that compared with that in healthy persons, the expression of Tim-3 on CD4 ${ }^{+}$T-lymphocytes and $\mathrm{CD}^{+} \mathrm{T}$-lymphocytes in the peripheral blood of patients with CHB and acute hepatitis B (AHB) is significantly increased and positively correlated with the severity of their disease. Further follow-up shows that after antiviral therapy, the condition of CHB patients enters the remission stage, whereas that of AHB patients enters the recovery stage. Tim-3 expression on $\mathrm{CD}^{+} \mathrm{T}$-lymphocytes and $\mathrm{CD} 8^{+} \mathrm{T}$-lymphocytes in the peripheral blood decreases significantly. These results indicate that the overexpression of Tim-3 is related to the occurrence and development of CHB and may be related to the prognosis of this disease. In addition, in vitro experiments confirm that the abilities of $\mathrm{CD}^{+} \mathrm{T}$-lymphocytes to proliferate and secrete antiviral cytokines in response to HBV antigen stimulation are significantly enhanced when the Tim-3 signaling pathway is blocked; these lymphocytes are derived from the peripheral blood of patients with CHB [17]. The findings of Nebbia et al. [18] also verified this conclusion. CHB is a familial aggregated infectious disease. Liao et al. [19] found that Tim-3 gene polymorphism is associated with the development of the disease after HBV infection. They speculated that a particular single-nucleotide polymorphism can progress into hepatocellular carcinoma or achieve hepatitis B surface antigen (HBsAg) serological conversion.

\section{Role of PD-1}

\subsection{Overview}

PD-1 is a type I transmembrane protein that is encoded by the Pdcd1 gene on chromosome 2, which is composed of 288 amino acids and expressed on activated T-lymphocytes as a receptor [20]. PD-1 was originally obtained from an apoptotic murine T-lymphocyte hybridoma through subtractive hybridization, and it is the 
first surface marker that can be used to identify impaired lymphocyte function. PD-1 belongs to the B7/CD28 superfamily and has two ligands, namely, PD-L1 and PD-L2. PD-L1, also known as B7-H1 or CD274, is a type I transmembrane protein encoded by the $C d 274$ gene of human chromosome 9, which is composed of 290 amino acids and expressed on antigen-presenting cells as well as tumor cells. PD-L1 plays an immunosuppressive effect when combined with PD-1 [21]. PD-L2, also known as B7-DC or CD273, can be expressed on dendritic cells and macrophages [22] and can inhibit T-lymphocyte activation [23]. The immunomodulatory effect of PD-1 in chronic viral infection was first found in the mouse lymphocytic choriomeningitis virus infection model. PD-1 was found to be highly expressed in the depleted CD8 ${ }^{+}$T-lymphocytes of the chronically infected mice. However, this phenomenon was not observed in the CD8 ${ }^{+}$T-lymphocytes with memory function in mice after virus elimination [24]. In addition, blocking the PD-1/PD-L1 signaling pathway in mice during in vivo experiments can increase the number of virus-specific $\mathrm{CD}^{+}$T-lymphocytes, enhance the cellular functions, and even interfere with virus replication [24]. By studying other chronic viral infections such as HIV infection, Zhang et al. [25] found that patients with disease progression have higher PD-1 expression in peripheral blood CD8 ${ }^{+}$T-lymphocytes and weaker cell function than those without disease progression. When the PD-1 signaling pathway is blocked, the ability of $\mathrm{CD} 8^{+} \mathrm{T}$-lymphocytes to secrete IFN-y is partially restored, which is associated with HIV susceptibility [26]. In chronic HCV infection, the expression of PD-1 in T-lymphocytes is associated with its antiviral efficacy [27]. PD-1 plays an important role in chronic viral infection and tumor immunity. High expression of PD-L1 can be detected in breast cancer, ovarian cancer, lung cancer, and other tumor tissues. In vivo and in vitro experiments also confirmed that PD-L1-transfected cells show weak antitumor invasion ability [28]. Recent clinical trials by some research institutions have predicted the potential value of anti-PD-1 drugs in tumor immunotherapy [29,30].

\subsection{PD-1 and chronic HBV infection}

Related literature shows that in chronic infections with viruses such as HIV [25,31,32] and HCV [33,34], the PD-1 signaling pathway plays an important immunomodulatory role in inhibiting the function of virus-specific $\mathrm{CD}^{+} \mathrm{T}$-lymphocytes. However, the immunomodulatory effect of PD-1 on chronic HBV infection remains unknown. Peng et al. [35] found that the PD-1 expression in HBV-specific CD8 ${ }^{+}$T-lymphocytes is significantly higher in CHB patients than in AHB patients and healthy persons, in addition to being positively correlated with HBV-DNA virus load in the serum. Germanidis et al. [36] recruited 53 CHB patients negative for the hepatitis B e antigen (HBeAg) (including 30 patients in the active phase of the disease and 23 patients with complete remission after long-term antiviral treatment) in their study, and the results show that the expression levels of forkhead transcription factor P3, PD-1, and PD-L1 in the liver tissues are significantly lower during remission than during the active stage. This finding indicated that PD-1 might be related to the prognosis of CHB. Another study found that in different stages of chronic HBV infection (immune tolerance, immune clearance, and virus carrier), PD-1 expression in $\mathrm{CD}^{+}$T-lymphocytes in the peripheral blood is higher than that in healthy persons. In addition, the increased PD-1 expression is induced by hepatitis B core antigen (HBcAg) through the C-jun N-terminal kinase (JNK), extracellular signal-regulated kinase (ERK), and phosphatidylinositol 3-kinase/protein kinase B (PI3K/AKT) signaling pathways [37]. Tzeng et al. [38] constructed an HBV-infected model mouse and found that the expression of PD-1 increases in the HBV-infected mouse with hepatic infiltrating lymphocytes, and that the HBV infection rate decreases when PD-1 is blocked. Raziorrouh et al. [39] cultured mononuclear cells from the peripheral blood of CHB patients in vitro and blocked the PD-1 signaling pathway. They found that the proliferative capacity of CD4+ $\mathrm{T}$-lymphocytes in the mononuclear cells from the above sources is reactivated, and its secretion of T helper $1\left(\mathrm{Th}_{1}\right)$-type cytokines, such as IFN- $y$ and tumor necrosis factor (TNF)- $\alpha$, also increases accordingly. Although the degree of upregulation of PD-1 expression in the peripheral blood or tissue cells of CHB patients varies in individuals and is related to the disease conditions, Ülger et al. [40] revealed, by using polymerase chain reaction-restriction fragment length polymorphism analysis, that PD-1 expression is not associated with $P D-1$ gene polymorphism. This finding is different from those of Li et al. [41], and the specific mechanism of upregulation of PD-1 expression needs further exploration. 


\section{Tim-3, PD-1, and chronic infection}

During chronic viral infection, including HBV infection, Tim-3 and PD-1 play a negative role in regulating the immune response. As immune molecules, these two might have a possible connection. Keir et al. [20], Sharpe et al. [22], and Crawford and Wherry [42] have suggested that blocking the PD-1/PD-L1 signaling pathway cannot fully restore the function of the "failed" T-lymphocytes. Other regulatory molecules play a negative role in regulating the function of T-lymphocytes. To confirm whether or not Tim-3 and PD-1 are coexpressed in $\mathrm{CD}^{+}{ }^{+}$-lymphocytes during chronic viral infection, Jin et al. [43] studied the expression levels of Tim-3 and PD-1 on virus-specific CD8 ${ }^{+}$T-lymphocytes in acute and chronic lymphocytic choroid plexus viral meningitis infection. They found that the levels in chronic infection are significantly higher than those in acute infection, and that the virus-specific CD8 ${ }^{+}$T-lymphocytes that coexpress Tim-3 and PD-1 show serious "functional failure". In virus-infected mice, simultaneously blocking the Tim-3 and PD-1 signaling pathways can significantly improve the immune response of $\mathrm{CD} 8^{+} \mathrm{T}$-lymphocytes and effectively control the virus; this process is more efficient than blocking either Tim-3 or PD-1 signaling pathway alone. Li et al. [41] determined Tim-3 and $P D-1$ gene polymorphisms in 845 chronic HBV-infected patients, 141 infected with HBV and self-recovered individuals, and 318 healthy persons through PCR. They found differences in the PD1 +8669 G/A and Tim3 -1516 G/T polymorphisms among the three groups. These sites are closely related to HBV-induced liver cirrhosis and liver cancer. These findings suggest that the expression levels of both PD-1 and Tim-3 increase in chronic HBV infection. Compared with those in healthy persons, the expression levels of Tim-3 and PD-1 on peripheral blood $\mathrm{CD}^{+} \mathrm{T}$-lymphocytes are higher in HBV-induced $\mathrm{CHB}$ and acute-on-chronic liver failure patients, especially those with acute-on-chronic liver failure [43]. Nebbia et al. [18] have verified that blocking the Tim-3/Gal-9 pathway based on the blocking of the PD-1 pathway in chronic HBV infection may play a complementary role in enhancing the immune response of HBV-specific T-lymphocytes. This finding suggests that these two pathways play a synergistic role in immunosuppression.

\section{Conclusion}

Tim-3 and PD-1 have immunosuppressive effects on chronic viral infection and play an important immunomodulatory effect on other chronic diseases, such as tumors and autoimmune diseases. To date, many studies have been conducted on the immunomodulatory effects of Tim-3 and PD-1 on chronic HBV infection. The overexpression of these two kinds of immune molecules affects the immune activity of immune cells and weakens their ability to clear the virus. Researchers should actively explore the targeted intervention of Tim-3 and PD-1 signaling pathways in chronic HBV infection, develop new drugs, and provide help for the clinical treatment of CHB.

Acknowledgments: None.

Conflict of interest: The author states no conflicts of interest.

\section{References}

[1] Ott J.J., Stevens G.A., Groeger J., Wiersma S.T., Global epidemiology of hepatitis B virus infection: new estimates of age-specific HBsAg seroprevalence and endemicity, Vaccine, 2012, 30(12), 2212-2219.

[2] Lozano R., Naghavi M., Foreman K., Lim S., Shibuya K., Aboyans V., et al., Global and regional mortality from 235 causes of death for 20 age groups in 1990 and 2010: a systematic analysis for the Global Burden of Disease Study2010, Lancet, 2012, 380(9859), 2095-2128.

[3] Sun H.H., Zhou D.F., Zhou J.Y., The role of DCs in the immunopathogenesis of chronic HBV infection and the methods of inducing DCs maturation, J. Med. Virol., 2016, 88(1), 13-20. 
[4] Park J.J., Wong D.K., Wahed A.S., Lee W.M., Feld J.J., Terrault N., et al., Hepatitis B virus-specific and global T-cell dysfunction in chronic hepatitis B, Gastroenterology, 2016, 150(3), 684-695.

[5] Zhang J., Feng G., Zhao Y., Zhang J., Feng L., Yang J., et al., Association between lymphocyte-to monocyte ratio (LMR) and the mortality of HBV-related liver cirrhosis: a retrospective cohort study, BMJ Open, 2015, 5(8), e008033.

[6] Rehermann B., Natural killer cells in viral hepatitis, Cell. Mol. Gastroenterol. Hepatol., 2015, 1(6), 578-588.

[7] McIntire J.J., Umetsu S.E., Akbari O., Potter M., Kuchroo V.K., Barsh G.S., et al., Identification of Tapr (an airway hyperreactivity regulatory locus) and the linked Tim gene family, Nat. Immunol., 2001, 2(12), 1109-1116.

[8] Meyers J.H., Sabatos C.A., Chakravarti S., Kuchroo V.K., The TIM gene family regulates autoimmune and allergic diseases, Trends Mol. Med., 2005, 11(8), 362-369.

[9] Golden-Mason L., Palmer B.E., Kassam N., Townshend-Bulson L., Livingston S., McMahon B.J., et al., Negative immune regulator Tim-3 is overexpressed on T cells in hepatitis C virus infection and its blockade rescues functional CD4+ and CD8+ T cells, J. Virol., 2009, 83(18), 9122-9130.

[10] de Kivit S., Lempsink L.J., Plants J., Martinson J., Keshavarzian A., Landay A.L., Modulation of TIM-3 expression on NK and T cell subsets in HIV immunological non-responders, Clin. Immunol., 2015, 156(1), 28-35.

[11] Cheng G., Li M., Wu J., Ji M., Fang C., Shi H., et al., Expression of Tim-3 in gastric cancer tissue and its relationship with prognosis, Int. J. Clin. Pathol., 2015, 8(8), 9452-9457.

[12] Wiener Z., Kohalmi B., Pocza P., Jeager J., Tolgyesi G., Toth S., et al., TIM-3 is expressed in melanoma cells and is upregulated in TGF-beta stimulated mast cells, J. Invest. Dermatol., 2007, 127(4), 906-914.

[13] Cai C., Zhang J., Li M., Wu Z.J., Song K.H., Zhan T.W., et al., Interleukin 10-expressing B cells inhibit tumor-infiltrating T cell function and correlate with T cell Tim-3 expression in renal cell carcinoma, Tumour Biol., 2016, 37(6), 8209-8218.

[14] Rong Y.H., Wan Z.H., Song H., Li Y.L., Zhu B., Zang H., et al., Tim-3 expression on peripheral monocytes and CD3+CD16/ CD56+natural killer-like T cells in patients with chronic hepatitis B, Tissue Antigens, 2014, 83(2), 76-81.

[15] Ju Y., Hou N., Meng J., Wang X., Zhang X., Zhao D., et al., T cell immunoglobulin and mucin domain-containing molecule-3 (Tim-3) mediates natural killer cell suppression in chronic hepatitis B, J. Hepatol., 2010, 52(3), 322-329.

[16] Wu W., Shi Y., Li J., Chen F., Chen Z., Zheng M., Tim-3 expression on peripheral T cell subsets correlates with disease progression in hepatitis B infection, Virol. J., 2011, 8, 113.

[17] Wu W., Shi Y., Li S., Zhang Y., Liu Y., Wu Y., et al., Blockade of Tim-3 signaling restores the virus-specific CD8(+) T-cell response in patients with chronic hepatitis B, Eur. J. Immunol., 2012, 42(5), 1180-1191.

[18] Nebbia G., Peppa D., Schurich A., Khanna P., Singh H.D., Cheng Y., et al., Upregulation of the Tim-3/galectin-9 pathway of T cell exhaustion in chronic hepatitis B virus infection, PLoS ONE, 2012, 7(10), e47648.

[19] Liao J., Zhang Q., Liao Y., Cai B., Chen J., Li L., et al., Association of T-cell immunoglobulin and mucin domain-containing molecule 3 (Tim-3) polymorphisms with susceptibility and disease progression of HBV infection, PLoS ONE, 2014, 9(5), e98280.

[20] Keir M.E., Butte M.J., Freeman G.J., Sharpe A.H., PD-1 and its ligands in tolerance and immunity, Annu. Rev. Immunol., 2008, 26, 677-704.

[21] Sharpe A.H., Freeman G.J., The B7-CD28 superfamily, Nat. Rev. Immunol., 2002, 2(2), 116-126.

[22] Sharpe A.H., Wherry E.J., Ahmed R., Freeman G.J., The function of programmed cell death 1 and its ligands in regulating autoimmunity and infection, Nat. Immunol., 2007, 8(3), 239-245.

[23] Latchman Y., Wood C.R., Chernova T., Chaudhary D., Borde M., Chernova I., et al., PD-L2 is a second ligand for PD-1 and inhibits T cell activation, Nat. Immunol., 2001, 2(3), 261-268.

[24] Blackburn S.D., Crawford A., Shin H., Polley A., Freeman G.J., Wherry E.J., Tissue-specific differences in PD-1 and PD-L1 expression during chronic viral infection: implications for CD8 T-cell exhaustion, J. Virol., 2010, 84(4), 2078-2089.

[25] Zhang J.Y., Zhang Z., Wang X., Fu J.L., Yao J., Jiao Y., et al., PD-1 up-regulation is correlated with HIV-specific memory CD8+ T cells exhaustion in typical progressors but not in long-term nonprogressors, Blood, 2007, 109(11), 4671-4678.

[26] Paris R.M., Petrovas C., Ferrando-Martinez S., Moysi E., Boswell K.L., Archer E., et al., Selective loss of early differentiated, highly functional PD-1 high CD4+ T cells with HIV progression, PLoS ONE, 2015, 10(12), e0144767.

[27] Salem M.L., El-Badawy A., Programmed death-1/programmed death-L1 signaling pathway and its blockade in hepatitis $C$ virus immunotherapy, World J. Hepatol., 2015, 7(23), 2449-2458.

[28] Dong H., Strome S.E., Salomao D.R., Tamura H., Hirano F., Flies D.B., et al., Tumor-associatedB7-H1promotes T-cell apoptosis: a potential mechanism of immune evasion, Nat. Med., 2002, 8(8), 793-800.

[29] Zitvogel L., Kroemer G., Targeting PD-1/PD-L1 interactions for cancer immunotherapy, Oncoimmunology, 2012, 1(8), 1223-1225.

[30] Curran M.A., Montalvo W., Yagita H., Allison J.P., PD-1 and CTLA-4 combination blockade expands infiltrating T cells and reduces regulatory T and myeloid cells with in B16 melanoma tumors, Proc. Natl. Acad. Sci. USA, 2010, 107(9), 4275-4280.

[31] Day C.L., Kaufmann D.E., Kiepiela P., Brown J.A., Moodley E.S., Reddy S., et al., PD-1 expression on HIV-specific T cells is associated with T-cell exhaustion and disease progression, Nature, 2006, 443(7109), 350-354.

[32] Petrovas C., Casazza J.P., Brenchley J.M., Price D.A., Gostick E., Adams W.C., et al., PD-1 is a regulator of virus-specific CD8 T cell survival in HIV infection, J. Exp. Med., 2006, 203(10), 2281-2292.

[33] Golden-Mason L., Palmer B., Klarquist J., Mengshol J.A., Castelblanco N., Rosen H.R., Upregulation of PD-1 expression on circulating and intrahepatic hepatitis $\mathrm{C}$ virus-specific $\mathrm{CD} 8^{+} \mathrm{T}$ cells associated with reversible immune dysfunction, J. Virol., 2007, 81(17), 9249-9258. 
[34] Urbani S., Amadei B., Tola D., Massari M., Schivazappa S., Missale G., et al., PD-1expressionin acute hepatitis C virus (HCV) infection is associated with HCV-specific CD8 exhaustion, J. Virol., 2006, 80(22), 11398-11403.

[35] Peng G., Li S., Wu W., Tan X., Chen Y., Chen Z., PD-1 upregulation is associated with HBV-specific T cell dysfunction in chronic hepatitis B patients, Mol. Immunol., 2008, 45(4), 963-970.

[36] Germanidis G., Argentou N., Hytiroglou P., Vassiliadis T., Patsiaoura K., Germenis A.E., et al., Liver FOXP3 and PD-1/PDL-1 expression is down-regulated in chronic HBV hepatitis on maintained remission related to the degree of inflammation, Front. Immunol., 2013, 4, 207.

[37] Li M., Sun X.H., Zhu X.J., Jin S.G., Zeng Z.J., Zhou Z.H., et al., HBcAg induces PD-1 upregulation on CD4+T cells through activation of JNK, ERK and PI3K/AKT pathways in chronic hepatitis-B-infected patients, Lab. Invest., 2012, 92(2), 295-304.

[38] Tzeng H.T., Tsai H.F., Liao H.J., Lin Y.J., Chen L., Chen P.J., et al., PD-1 blockage reverses immune dysfunction and hepatitis B viral persistence in a mouse animal model, PLoS ONE, 2012, 7(6), e39179.

[39] Raziorrouh B., Heeg M., Kurktschiev P., Schraut W., Zachoval R., Wendtner C., et al., Inhibitory phenotype of HBV-specific CD4+ T-cells is characterized by high PD-1 expression but absent coregulation of multiple inhibitory molecules, PLoS ONE, 2014, 9(8), e105703.

[40] Ülger Y., Bayram S., Sandıkçı M.Ü, Akgöllü E., Bekar A., Relationship between programmed cell death-1 polymorphisms and clearance of hepatitis B virus, Int. J. Immunogenet., 2015, 42(3), 133-139.

[41] Li Z., Li N., Zhu Q., Zhang G., Han Q., Zhang P., et al., Genetic variations of PD1 and TIM3 are differentially and interactively associated with the development of cirrhosis and HCC in patients with chronic HBV infection, Infect. Genet. Evol., 2013, 14, 240-246.

[42] Crawford A., Wherry E.J., The diversity of costimulatory and inhibitory receptor pathways and the regulation of antiviral T cell responses, Curr. Opin. Immunol., 2009, 21(2), 179-186.

[43] Jin H.T., Anderson A.C., Tan W.G., West E.E., Ha S.J., Araki K., et al., Cooperation of Tim-3 and PD-1 in CD8 T-cell exhaustion during chronic viral infection, Proc. Natl. Acad. Sci. USA, 2010, 107(33), 14733-14738. 